\title{
Inventario del Desarrollo de Habilidades Comunicativas McArthur-Bates (CDI): propuesta de una versión abreviada*
}

\section{McArthur-Bates Communicative Development Inventory (CDI): Proposal of an Abbreviate Version}

Recibido: enero 14 de 2009 | Revisado: noviembre 10 de 2009 | Aceptado: agosto 2 de 2010

\author{
ChAMARRITA FARKAS ** \\ Pontificia Universidad Católica de Chile
}

Para citar este artículo. Farkas, C. (2011). Inventario del Desarrollo de Habilidaddes Comunicativas McArthur-Bates (CDI): propuesta de una versión abreviada. Universitas Psychologica, 10(1), 245-262.

* Artículo de investigación que contó con la financiación otorgada por el Fondo Nacional de Desarrollo Científico y Tecnológico, FONDECYT, № 1060778.

** Escuela de Psicología, Pontificia Universidad Católica de Chile, Ave. Vicuña Mackenna 4860, Macul, Santiago, Chile. Teléfono: 56-2-3547067. Fax: 562-3544844.Correo electrónico: chfarkas@uc.cl

\section{RESUMEN}

Los Inventarios del Desarrollo de Habilidades Comunicativas de McArthurBates (CDI) evalúan el desarrollo del lenguaje en niños y niñas, a través del reporte de un cuidador significativo. El primer inventario evalúa lenguaje verbal y no verbal en infantes de 8 y 18 meses y se compone de 949 reactivos, distribuidos en 6 escalas. Este estudio propone una versión abreviada del instrumento, para lo cual fue aplicado a familiares y educadoras de 130 infantes chilenos de 11 a 15 meses. Se realizaron análisis de reactivos, de confiabilidad y validez, y análisis factorial de las subescalas. La versión abreviada considera 241 reactivos, distribuidos en 4 escalas. Las evaluaciones de las propiedades psicométricas de la escala resultaron satisfactorias, mostrando confiabilidad y validez adecuadas.

Palabras clave autor

Lenguaje, instrumento abreviado, psicometría, reporte de cuidadores.

Palabras clave descriptores

Desarrollo infantil, desarrollo del lenguaje, psicometría.

\section{A B S T R ACT}

The McArthur-Bates Communicative Development Inventories (CDI) assesses language development en children, through a significant caregiver report. The first inventory assesses verbal and non verbal language in infants who are from 8 to 18 months old and it is composed of 949 items distributed in 6 scales. This study proposes an abbreviate form of this instrument, and was tested on families and educators of 130 Chilean children of 11-15 months old. Analyses related to the items, reliability and validity of the instrument and factorial analyses of subscales were realized. The abbreviate version consider 241 items distributed in 4 scales. The evaluation of the psychometric properties of the instrument was acceptable, demonstrating adequate reliability and validity.

Key words author

Language, Abbreviate Instrument, Psychometric, Caregivers Report.

Key words plus

Child Development, Language Developmet, Psychometrics. 
Los Inventarios del Desarrollo de Habilidades Comunicativas de MacArthur-Bates (CDI) fueron desarrollados por Larry Fenson (Fenson et al., 1994) y adaptados al español por Jackson-Maldonado, Bates y Thal (Jackson-Maldonado, Thal, Fenson, Marchman, Newton \& Conboy, 2003). Su objetivo es evaluar el desarrollo de habilidades comunicativas en niños y niñas, a través del reporte de un cuidador significativo.

La versión original de estos inventarios se desarrolló solo con propósitos de investigación por Bates, Camaioni y Volterra (1975). Su objetivo era obtener información de las habilidades comunicativas tempranas en los niños, y los instrumentos existentes solo permitían realizar screening, por lo que la información ofrecida no permitía discriminar adecuadamente. En el estudio original realizado en 1975, se utilizó una metodología combinada entre el reporte de los padres y grabaciones en videos de las conductas comunicativas de los niños, encontrando que el reporte de los padres era un excelente predictor de la evaluación de estas conductas comunicativas en los niños. La ventaja de los inventarios creados era que además implicaban un menor trabajo en su aplicación y posterior codificación en relación con otros métodos, y que no había otros instrumentos para evaluar habilidades comunicativas en niños menores de 2 años.

El desarrollo de estos inventarios se basó en la consideración de otros instrumentos similares (p. ej., un inventario italiano desarrollado por Luchenti, Osella, Tieri \& Volterra, 1988 citados en Fenson et al., 1994), así como evaluaciones y estudios previos sobre el tema, realizados por los autores (Bates, Benigni, Bretherton, Camaioni \& Volterra, 1979; Bates, Bretherton \& Snyder, 1988; Bates, Camaioni \& Volterra, 1975; Fenson, Thal \& Bates, 1990; Uzgiris \& Hunt, 1975).

El CDI entrega información sobre el desarrollo de habilidades comunicativas en niños y niñas, a través del reporte de un cuidador significativo (madre, padre, educadora u otro). Se compone de 2 inventarios: "Primeras palabras y gestos" (versión W \& G o "Word and Gestures" en inglés; 8 a 18 meses de edad), y "Palabras y enunciados" (versión
W \& S; 16 a 30 meses). Para este artículo se considerará la forma en español del primer inventario.

La versión final del inventario para infantes (W \& G) quedó compuesta de dos partes. La primera, enfocada al lenguaje verbal, se compone de 3 escalas. La primera escala aborda el inicio del lenguaje verbal en el niño, indagando su comprensión de distintas frases o sonidos iniciales. Las segunda y tercera, lenguaje comprensivo y expresivo, pretenden evaluar a través del reporte del adulto, si las palabras consultadas son primero comprendidas, y luego producidas por el niño(a). Dichas palabras se encuentran categorizadas en 22 categorías semánticas basadas en listas de vocabulario que consideran sustantivos, personas, verbos, adjetivos, pronombres, proposiciones, locativos y cuantificadores.

La segunda parte se focalizó en un rango de habilidades comunicativas y representacionales tempranas, que no dependen de la expresión verbal. Para ello, se consideraron gestos tempranos que son indicadores de intención comunicativa, previo al desarrollo del lenguaje verbal (Bates et al., 1975), gestos que se consideran como un indicador temprano de habilidad simbólica, así como un hito previo del desarrollo lingüístico (Acredolo \& Goodwyn, 1985, 1988; Bates et al., 1979; Piaget, 1962), y gestos que son útiles para evaluar habilidades comunicativas y simbólicas en infantes con pobre lenguaje expresivo o con déficits ((Rescorla \& Goossens, 1992; Terrell \& Schwartz, 1988; Terrell, Schwartz, Prelock, \& Messick, 1984; Thal \& Tobias, 1993). Esta parte se refiere a la evaluación de los gestos tempranos y tardíos, entendiéndose por gestos tempranos a aquellos que indican intención comunicativa, así como juegos y rutinas con adultos que indican función simbólica. Los gestos tardíos se refieren a la realización de gestos y acciones a los que subyace función simbólica (juegos de "como si").

En síntesis, la versión $W \&$ G tiene como objetivo evaluar aspectos comprensivos (primeras frases y vocabulario) y expresivos (vocabulario) del lenguaje verbal, así como aspectos del lenguaje no verbal (gestos tempranos y tardíos). 


\section{Población destinataria}

La población destinataria del CDI (versión W \& $\mathrm{G})$ son niños y niñas entre 8 y 18 meses de edad. Su traducción a distintos idiomas en distintos países y su construcción de normas en distintas poblaciones, lo propone como un instrumento que puede ser aplicado a niños de distintas culturas.

La versión en español que se usó para este estudio tiene como población destinataria niños de habla hispana como lengua materna. Se puede aplicar tanto a niños con un desarrollo de lenguaje normal, como a aquellos que presentan un cierto retraso, con el objetivo de pesquisar, diagnosticar o evaluar los efectos de una intervención.

\section{Características generales del inventario original $W \& G$}

Este inventario evalúa, a través de la percepción y reporte del adulto, el entendimiento del niño de ítems de vocabulario separados en categorías semánticas, y considera si la palabra es comprendida y producida. Luego, consulta sobre gestos comunicativos y simbólicos realizados por el niño. Considera 949 reactivos, distribuidos en 6 escalas, las cuales se presentan en el siguiente cuadro.

\section{CuAdro 1}

Escalas del CDI forma $W \& G$

\begin{tabular}{lc}
\hline \multicolumn{1}{c}{ chfarkas@uc.c } & № de reactivos \\
\hline Comprensión de primeras frases & 28 \\
Lenguaje comprensivo & 428 \\
Lenguaje expresivo & 428 \\
Gestos tempranos & 21 \\
Gestos tardíos & 44 \\
Gestos totales $(*)$ &
\end{tabular}

(*) Esta escala no tiene reactivos adicionales; constituye la suma de los puntajes en las dos escalas anteriores.

Fuente: basado en Fenson et al. (1994).

La primera escala aborda el inicio del lenguaje verbal en el niño. Las segunda y tercera, lenguaje comprensivo y expresivo, se refieren respectivamente a la comprensión y producción de distintas palabras. La cuarta escala consulta la realización de gestos tempranos que indican intención comunicativa, así como juegos y rutinas con adultos que indican función simbólica. Por último, la quinta escala se refiere a la realización de gestos y acciones a los que subyace función simbólica (juegos de "como si"). La sexta escala es la suma de las dos escalas anteriores.

La primera escala no se subdivide en subescalas. Tanto la escala de Lenguaje Expresivo como la de Lenguaje Comprensivo se dividen en 22 subescalas, que corresponden a categorías semánticas. La escala de Gestos Tempranos se compone de 2 subescalas y la de Gestos Tardíos, de 4. El siguiente cuadro ilustra las escalas con sus respectivas subescalas.

\section{Propiedades de la administración y la puntuación de la versión original}

El CDI es un inventario de autoaplicación. Se puede aplicar de manera individual o grupal, y solo requiere del inventario y un lápiz. Se considera que su aplicación dura aproximadamente 30 minutos, lo cual varía de acuerdo con el nivel cognitivo y de comprensión de la persona que contesta. No obstante ser un inventario autoaplicado, cuando se usa en poblaciones de nivel socioeconómico (NSE) bajo, es recomendable que haya un evaluador junto al adulto que contesta, que apoye la adecuada comprensión de las instrucciones.

Cada reactivo se puntúa en términos de Presencia (1) o Ausencia (0) ${ }^{1}$. A través de la suma de todos los reactivos, se obtiene un puntaje bruto para cada una de las seis escalas. Luego, cada uno de estos puntajes brutos, en función de la edad del niño, se transforma en un percentil. Todo este procedimiento se realiza con un software especialmente diseñado para la puntuación de la prueba, cuyo objetivo es disminuir los errores que ocurren en la tabulación, facilitar el trabajo y estandarizar el reporte de los resultados.

1 Excepto la subescala "primeros gestos" de la escala de Gestos Tempranos, que considera 3 categorías de respuesta. 
CuAdro 2

Escalas y subescalas del CDI forma W \& G

\begin{tabular}{llrlr}
\hline \multicolumn{1}{c}{ Escalas } & \multicolumn{1}{c}{ Subescalas } & No reactivos & Subescalas & № reactivos \\
\hline Lenguaje & Sonidos de cosas y animales & 12 & Rutina diaria, reglas sociales y juegos & 19 \\
Expresivo y & Animales & 36 & Acciones y procesos & 55 \\
Comprensivo & Vehículos & 9 & Estados & 2 \\
& Alimentos y bebidas & 30 & Tiempo & 8 \\
& Ropa & 19 & Cualidades y atributos & 37 \\
& Partes del cuerpo & 20 & Pronombre & 33 \\
& Juguetes & 8 & Preguntas & 6 \\
& Utensilios de la casa & 36 & Artículos & 8 \\
& Muebles y cuartos & 24 & Cuantificadores y adverbios & 8 \\
Gestos tempra- & Lugares y objetos (fuera casa) & 26 & Locativos & 9 \\
nos & Personas & 20 & Preposiciones & 3 \\
& Primeros gestos & 13 & Juegos con adultos y rutinas & 8 \\
Gestos tardíos & Acciones con objetos & 15 & Imitación de otros tipos de actividades & 15 \\
& Jugar a ser adulto & 13 & de adultos & \\
& & & Acciones con un objeto en vez de otro & 1 \\
\hline
\end{tabular}

Fuente: basado en Foster et al. (1994).

\section{Propiedades psicométricas del inventario $\mathrm{W}$ $\mathcal{E} \mathrm{G}$ (versión original)}

Los análisis de confiabilidad del inventario W \& $\mathrm{G}$ (versión en inglés) arrojaron en una muestra de 659 niños americanos, un alfa de Cronbach de 0.95 para vocabulario comprensivo y 0.96 para lenguaje expresivo. Para la parte de gestos y acciones se realizaron análisis correlacionales debido al bajo número de subescalas, obteniéndose correlaciones entre 0.45 y 0.85 , todas ellas significativas. Se realizó un análisis test-retest con una muestra de 137 niños, obteniéndose correlaciones de Pearson de $0.87,0.95$ y 0.86 (con un $p<0.01$ ) para comprensión, expresión y gestos, respectivamente (Jackson-Maldonado et al., 2003) .

Se realizaron distintos estudios de validez concurrente con mediciones de laboratorio (observaciones) y otros inventarios, como por ejemplo el Expressive One Word Picture Vocabulary Test (EOWPVT), obteniéndose rangos de correlación entre .33 y .85 (media de 0.61) (Jackson-Maldonado et al., 2003).

La versión completa en español del inventario $W \& G$ (que es la que se pretende abreviar en este estudio) fue analizada en una muestra de 778 ni- ños mexicanos, de ambos sexos, de distintos NSE y que abarcaban todas las edades consideradas por el instrumento. El análisis muestra una alta confiabilidad interna, obteniéndose un alfa de Cronbach de 0.94 para vocabulario comprensivo, 0.94 para lenguaje expresivo, y 0.89 para gestos totales (que abarca a las dos escalas de gestos y acciones) (Jackson-Maldonado et al., 2003). Se realizó además un análisis test-retest con 16 familias, dentro de un lapso de un mes. Se obtuvieron correlaciones de $0.97(p<0.001)$ para la parte comprensiva y 0.81 $(p<0.001)$ en la parte expresiva o de producción (Jackson-Maldonado et al., 2003).

Para la validez de contenido, se contrastó el inventario con: base de datos de disertaciones doctorales, base de datos de lenguaje español no publicada, otros ítems de evaluación de lenguaje en español, y los comentarios al instrumento de un grupo de padres. Para la validez concurrente, se compararon los resultados del inventario con la observación espontánea del lenguaje y juego, de una muestra de 19 niños de 10 meses y 20 niños de 12 meses. La correlación obtenida en la parte productiva del inventario fue moderadamente fuerte $(r=0.61, p<0.005)$ y moderada para la parte de los gestos $(r=0.47, p<0.04)$, en los niños de 12 
meses de edad. No hubo suficientes datos con los niños de 10 meses así como en la expresión espontánea del lenguaje, para poder hacer los análisis (Jackson-Maldonado et al., 2003).

En cuanto a la validez predictiva, se han encontrado correlaciones en el rango de .60 a .80 en la producción de lenguaje, usando el reporte de los padres a los 20 meses de edad, y con varias mediciones de vocabulario y gramática a los 28 meses de edad, usando datos observacionales. La validez predictiva de las secciones de vocabulario se obtuvo a través de estudios con la penúltima versión del inventario, encontrándose correlaciones entre 0.70 y 0.97 ( $p<0.001$ ) (Jackson-Maldonado et al., 2003).

Este inventario cuenta con una primera adaptación a la población chilena, realizada por la autora de este estudio. En esta adaptación se verificó el instrumento por parte de un grupo de 5 jueces, quienes revisaron la adecuación del vocabulario empleado para la población chilena, así como la claridad y adecuación de las instrucciones. Además, se aplicó a una muestra piloto de 40 madres, como potenciales evaluadas, para chequear su comprensión del vocabulario, los conceptos y la claridad de las instrucciones. Pese a ser una medición indirecta del lenguaje del niño y no contar con normas para niños chilenos, hay que considerar que no existen tests de lenguaje para medir directamente a los niños antes de los dos años. Además, estos inventarios están ampliamente avalados por investigaciones internacionales y especializadas en el tema.

El objetivo de este estudio fue analizar este inventario en una muestra de niños y niñas chilenos, de modo que se pudiera proponer una versión abreviada del mismo, que contara con buenas propiedades psicométricas así como índices adecuados de confiabilidad y validez. Se pretende que esta versión abreviada sea una herramienta útil, a la hora de contar con un instrumento que permita discriminar adecuadamente poblaciones distintas así como el efecto de una intervención, favoreciendo un tiempo de respuesta más reducido y disminuyendo el efecto de variables motivacionales y de cansancio.

\section{Método}

\section{Descripción de la muestra y recolección de los datos}

La muestra a la que se aplicó este inventario estuvo compuesta por 130 niños y niñas entre 11 y 15 meses (media 12.29, DS = 0.858). Su NSE corresponde a un NSE bajo, distribuyéndose en 3 categorías de pobreza (evaluación realizada por la asistente social de la institución): no pobreza, pobreza y pobreza-indigencia. Todos los niños asistían a salas cuna de una institución dependiente del Estado, distribuyéndose en 20 salas cuna distintas, las cuales corresponden a distintas comunas de la ciudad de Santiago de Chile.

La selección de dicha muestra se justifica en el hecho de que, actualmente en Chile, se están realizando una serie de intervenciones para paliar los efectos que tiene para los niños el nacer en un contexto psicosocial adverso, y así disminuir la brecha de la inequidad social. Los esfuerzos del Gobierno se han centrado, así, en programas orientados a la primera infancia así como aumentar la cobertura de salas cuna y jardines infantiles, pero no se cuenta con instrumentos que permitan evaluar adecuadamente estas intervenciones. Por tanto, el objetivo de crear una versión abreviada y útil del inventario CDI está pensado para ser aplicado a este tipo de población. Es decir, infantes de NSE bajo y que asisten a sala cuna, de modo que pueda ser utilizado no solo con las familias, sino con el personal educativo. Aunque ello puede llevar a que el uso del inventario abreviado deba ser usado con cautela en otros niveles sociales, éstos sí debieran ser considerados en estudios futuros con esta versión abreviada, al momento de generar normas y estandarizarla.

Además, de los 130 niños evaluados, algunos han estado sometidos a una intervención que potencia el uso de gestos simbólicos, por un tiempo aproximado de 4 meses, mientras que los niños restantes no. El que los niños estuviesen sometidos a una intervención se debe a que conformaban parte de un proyecto cuyo objetivo era evaluar los efectos de potenciar la comunicación gestual sobre 
el desarrollo de la comunicación verbal, efectos que fueron evaluados con la versión abreviada del CDI, propuesta en este estudio. Para los análisis psicométricos de la prueba se utilizaron ambos grupos de niños (con/sin intervención) de modo que se aumentara la heterogeneidad de la muestra, así como se ayudara a encontrar los reactivos que mejor permitieran discriminar los efectos de la intervención realizada.

El inventario fue respondido por un familiar significativo del niño que estaba a cargo de su cuidado diario y por un cuidador educativo significativo (educadora de párvulos o técnica auxiliar de párvulos). De los familiares que respondieron el CDI, un 95\% fueron las madres de los niños y el
$5 \%$ restante, sus abuelas o padres. Para diferenciar esta aplicación de la realizada a las educadoras, se seguirá refiriendo genéricamente a "los padres".

Previamente a la aplicación del instrumento, se les explicaba a los padres que la participación en el estudio era voluntaria, y se les aseguraba que los datos serían considerados de manera anónima. Los que asentían a participar del estudio firmaban una hoja de consentimiento y luego el evaluador les daba las instrucciones y velaba por su adecuado entendimiento, mientras éstos respondían por sî mismos los ítems.

En la Tabla 1 se aprecia la distribución de la muestra considerando sexo, edad, sala cuna de pertenencia, NSE y grupo con/sin intervención.

\section{TABLA 1}

Distribución de la muestra por sexo, sala cuna, intervención, edad y NSE

\begin{tabular}{|c|c|c|c|c|c|c|}
\hline & & Frecuencia & Porcentaje & \multicolumn{2}{|c|}{ Frecuencia } & Porcentaje \\
\hline \multirow[t]{3}{*}{ SEXO } & 1 & 74 & 56.9 & & & \\
\hline & 2 & 56 & 43.1 & & & \\
\hline & Total & 130 & 100.0 & & & \\
\hline \multirow[t]{11}{*}{ SALA CUNA } & 1 & 10 & 7.7 & 11 & 3 & 2.3 \\
\hline & 2 & 7 & 5.4 & 12 & 2 & 1.5 \\
\hline & 3 & 10 & 7.7 & 13 & 6 & 4.6 \\
\hline & 4 & 7 & 5.4 & 14 & 4 & 3.1 \\
\hline & 5 & 10 & 7.7 & 15 & 9 & 6.9 \\
\hline & 6 & 7 & 5.4 & 16 & 4 & 3.1 \\
\hline & 7 & 10 & 7.7 & 17 & 2 & 1.5 \\
\hline & 8 & 3 & 2.3 & 18 & 9 & 6.9 \\
\hline & 9 & 6 & 4.6 & 19 & 4 & 3.1 \\
\hline & 10 & 12 & 9.2 & 20 & 5 & 3.8 \\
\hline & Total & 130 & 100.0 & & & \\
\hline \multirow[t]{3}{*}{ CON/SIN INT. } & 1 & 80 & 61.5 & & & \\
\hline & 2 & 50 & 38.5 & & & \\
\hline & Total & 130 & 100.0 & & & \\
\hline \multirow[t]{2}{*}{ EDAD } & 11 & 14 & 10.8 & 14 & 8 & 6.2 \\
\hline & 12 & 80 & 61.5 & 15 & 4 & 3.1 \\
\hline
\end{tabular}




\begin{tabular}{|c|c|c|c|c|c|c|}
\hline & & & Frecuencia & Porcentaje & Frecuencia & Porcentaje \\
\hline & & 13 & 24 & 18.5 & & \\
\hline & & Total & 130 & 100.0 & & \\
\hline \multirow[t]{6}{*}{ NSE } & Válidos & 1 & 28 & 24.8 & & \\
\hline & & 2 & 49 & 43.4 & & \\
\hline & & 3 & 36 & 31.9 & & \\
\hline & & Total & 113 & 100.0 & & \\
\hline & Perdidos & Sistema & 17 & & & \\
\hline & Total & 130 & 100.0 & & & \\
\hline
\end{tabular}

$N=130$

Sexo: 1: varón, 2: niña: sala cuna de 1 a 20; Intervención: 1: con, 2: sin; Edad: 11 a 15 meses; NSE: 1: pobreza indigencia, 2: pobreza, 3: no pobreza.

Fuente: elaboración propia.

\section{Procedimientos estadísticos}

Este estudio fue diseñado para realizar el análisis discriminativo de los ítems y así seleccionar los mejores reactivos que conformarían la versión abreviada del inventario. Para ello, se analizó primero la confiabilidad de la escala total, a través del coeficiente alfa de Cronbach. Luego, se procedió a analizar todos los reactivos del CDI (versión padres) con el software ITEMAN, que es un programa especializado en el análisis de reactivos de acuerdo con la Teoría de la respuesta a la prueba, ofreciendo información útil acerca de los niveles de dificultad de cada reactivo, la discriminación entre desempeños altos y bajos, la distribución de respuestas en las opciones o distractores de cada reactivo, la correlación entre la respuesta al reactivo con respecto a las respuestas a toda la prueba, entre otros. Se optó por utilizar este tipo de análisis, considerando el tamaño muestral así como la alta correlación que éste tiene con el Análisis de Respuesta al Ítem (correlación de 0.80). El criterio de selección de los reactivos fue que cumplieran con los siguientes requisitos: (a) grado de dificultad en un rango de 0.2 a 0.8 , con un promedio cercano a 0.5 , con lo que se pretende seleccionar aquellos reactivos que, para esta muestra, presenten adecuados índices de discriminabilidad y que la dis- tribución de los puntajes de los sujetos sea lo más normal posible; (b) correlación biserial puntual igual o superior a 0.3 ; (c) distribución homogénea entre los distractores y (d) porcentaje de omisiones no mayor a un $20 \%$.

Posteriormente, se procedió a analizar la confiabilidad de la escala abreviada propuesta, a través del coeficiente alfa de Cronbach, para analizar la consistencia interna de la misma.

Se revisaron las escalas de vocabulario comprensivo, vocabulario expresivo y gestos totales con un análisis factorial a través del método de análisis de los componentes principales con una rotación Varimax de Kaiser, para ver cómo se agrupaban de mejor manera los reactivos. No se analizó la escala de comprensión de primeras frases ya que ésta no se subdivide en subescalas, y ya que la escala de gestos totales se compone de dos subescalas (gestos tempranos y gestos tardíos), se optó por tomar la escala más general para su análisis. Finalmente, para analizar la validez de constructo de la versión abreviada, se obtuvo evidencia correlacional a través de un análisis entre escalas y subescalas, además de evidencia convergente, a través de un análisis comparativo entre las respuestas de padres y educadoras, y correlacionando las evaluaciones realizadas por padres y educadoras, con los resultados en la escala de lenguaje del test Bayley III 
(Bayley, 2006), el cual fue aplicado a una submuestra de 62 niños.

Posteriormente, cuando todos los niños de la muestra cumplieron los 18 meses de edad, se les volvieron a aplicar los instrumentos, con el fin de ver si mejoraban las correlaciones entre padres y educadoras, y respecto al Bayley III.

\section{Resultados}

\section{Análisis de Confiabilidad de la Versión Original del Inventario CDI (W Eु G)}

Tanto el inventario respondido por los padres así como por las educadoras obtuvo buenos índices de confiabilidad, oscilando entre 0.885 y 0.990 , aunque cabe considerar que no todas las escalas permitieron realizar los análisis por el bajo índice de respuestas (versión educadoras, software SPSS). Los resultados de la consistencia interna de las escalas se encuentran en la Tabla 2.

TABLA 2

Análisis de la confiabilidad para las escalas del CDI original

\begin{tabular}{|c|c|c|}
\hline Escala & $\begin{array}{l}\text { Aplicación } \\
\text { padres }\end{array}$ & $\begin{array}{l}\text { Aplicación } \\
\text { educadoras }\end{array}$ \\
\hline $\begin{array}{l}\text { 1. Comprensión primeras } \\
\text { frases }\end{array}$ & 0.885 & 0.921 \\
\hline 2. Vocabulario comprensivo & 0.990 & $(*)$ \\
\hline 3. Vocabulario expresivo & 0.916 & $(*)$ \\
\hline 4. Gestos tempranos & 0.771 & 0.899 \\
\hline 5. Gestos tardíos & 0.917 & 0.890 \\
\hline
\end{tabular}

(*)La escala tiene menos de dos elementos con varianza diferente de cero.

(**) No se incluyó la escala 6 (gestos totales), por ser ésta solamente la suma de las escalas 4 y 5 .

Fuente: elaboración propia.

\section{Análisis de los Reactivos de la Versión Original del CDI}

Se procedió a analizar todos los reactivos del CDI (versión padres) con el objetivo de seleccionar los mejores, en cuanto a sus características y capacidad discriminativa. Una vez realizados los análisis, se seleccionaron 241 reactivos, de los 949 originales que componían el inventario.

Para todas las escalas, se seleccionaron solo los reactivos que cumplían con los requisitos de ser un "buen ítem" (ver descripción de criterios en apartado procedimientos estadísticos), excepto para la escala de Vocabulario Expresivo, en la cual la cantidad de ítems que cumplían dicho criterio era muy baja (10) lo cual reducía notoriamente su confiabilidad, por lo cual se amplió la selección a los "items regulares", 2 quedando finalmente la escala compuesta por 19 ítems.

Por último, se decidió considerar solamente la escala Gestos Totales, sin dividirla en las escalas Gestos Tempranos y Gestos Tardíos, quedando la forma abreviada del instrumento en 4 escalas. La Tabla 3 muestra la distribución de reactivos en la versión original y la versión abreviada propuesta para el inventario.

TABLA 3

Distribución de los reactivos para las escalas del CDI, versiones original y abreviada

\begin{tabular}{lcc}
\hline \multicolumn{1}{c}{ Escala } & $\begin{array}{c}\text { № reactivos } \\
\text { versión } \\
\text { original }\end{array}$ & $\begin{array}{c}\text { № reactivos } \\
\text { versión } \\
\text { abreviada }\end{array}$ \\
\hline $\begin{array}{l}\text { Comprensión primeras } \\
\text { frases }\end{array}$ & 28 & 19 \\
Vocabulario comprensivo & 428 & 165 \\
Vocabulario expresivo & 428 & 19 \\
Gestos tempranos & 21 & \\
Gestos tardíos & 44 & 38 \\
Gestos totales(*) & 65 & 241 \\
TOTAL & 949 & \\
\hline
\end{tabular}

(**) La escala 6 (gestos totales), se constituye por la suma de las escalas 4 y 5 . Por tal motivo no es incluida en la suma de los reactivos totales.

Fuente: elaboración propia con base en Foster et al. (1994).

2 Aquellos que no cumplían con todos los requisitos antes expuestos, privilegiándose el grado de dificultad y la correlación biserial puntual. 


\section{Análisis de confiabilidad de la versión abreviada del Inventario CDI}

El análisis de la confiabilidad de la versión abreviada en la aplicación a padres, muestra buenos índices de confiabilidad, los cuales se ubican en un rango de .841 a .982. De acuerdo con Nunnally (1978) lo recomendable es que la confiabilidad se encuentre sobre el promedio de 0.70 , encontrándose que todas las escalas evaluadas de la versión abreviada superan este criterio.

En la Tabla 4, se presenta una comparación entre la confiabilidad de las versiones original y abreviada de las escalas del CDI. Como se puede apreciar, la versión abreviada del instrumento muestra, en general, homogeneidad en sus niveles de confiabilidad en las distintas escalas que lo constituyen, presentando las escalas finales una buena confiabilidad.

Con posterioridad, se realizó el análisis de confiabilidad a la versión abreviada de los CDI respondidos por las educadoras. La Tabla 4 presenta dicha información. Como se puede advertir, la confiabilidad de las escalas sigue siendo buena, oscilando entre .837 y .981 , e incluso puede, en esta oportunidad, obtenerse información sobre las escalas que no habían podido ser analizadas sobre la base del instrumento completo.

\section{Análisis factorial de las escalas de la versión abreviada del Inventario CDI}

Este análisis pretendió apreciar cómo se agrupaban los reactivos de mejor manera, y si se mantenían las subescalas propuestas por la forma original del CDI (categorías semánticas). Este análisis no se realizó para la Escala Comprensión de Primeras Frases, ya que desde el inicio ésta no estaba compuesta por subescalas, prefiriéndose por tanto mantenerla igual.

\section{Análisis factorial para Escala Vocabulario Comprensivo}

El análisis factorial para esta escala no permite obtener valores de las medidas de adecuación muestral de Kaiser-Meyer-Olkin (KMO) y de la esfericidad de Bartlett, ya que la matriz no es definida como positiva. Originalmente el análisis arrojó 44 factores, que explicaban el $82.7 \%$ de la varianza. Con el objetivo de reducir la cantidad de factores y que a su vez éstos incluyeran todos los reactivos, se fueron ensayando distintas soluciones, hasta llegar a 4 factores (que contenían los pesos factoriales más altos de los reactivos), los cuales explican un $36.6 \%$ de la varianza en las respuestas. La Tabla 5 muestra dichos factores con el nombre propuesto,

\section{TABLA 4}

Comparación confiabilidad para las escalas de la versión original y abreviada del CDI, versión padres y versión educadoras

\begin{tabular}{lllll}
\hline \multicolumn{1}{c}{ Escala } & \multicolumn{2}{c}{ Padres } & \multicolumn{2}{c}{ Educadoras } \\
& Versión original & Versión abreviada & Versión original & Versión abreviada \\
\hline 1. Comprensión Primeras Frases & 0.885 & 0.875 & 0.921 & 0.907 \\
2. Vocabulario Comprensivo & 0.990 & 0.982 & $\left(^{*}\right)$ & 0.981 \\
3. Vocabulario Expresivo & 0.916 & 0.841 & $\left(^{*}\right)$ & 0.837 \\
4. Gestos tempranos & 0.771 & & 0.899 & \\
5. Gestos tardíos & 0.917 & & 0.890 & \\
4. Gestos Totales & 0.931 & 0.905 & 0.930 & 0.898 \\
\hline
\end{tabular}

(*) La escala tiene menos de dos elementos con varianza diferente de cero.

Fuente: elaboración propia con base en Foster et al. (1994). 
el número de ítems que consideran y el porcentaje de varianza explicada.

El Factor 1 agrupa 32 reactivos y se refiere a la comprensión de un vocabulario inicial de tipo cotidiano para el niño. El Factor 2 considera 41 ítems y hace alusión a la comprensión de un vocabulario inicial, pero menos cotidiano para el niño. El Factor 3 agrupa 36 reactivos y se refiere a la comprensión de un vocabulario avanzado de tipo cotidiano. Finalmente el Factor 4 incluye 49 ítems y hace alusión a la comprensión de un vocabulario avanzado de tipo gramatical. En síntesis, puede apreciarse que los factores que agrupan a los ítems se basan en dos criterios: grado de dificultad de las palabras y nivel de cotidianeidad del lenguaje.

\section{Análisis factorial para Escala Vocabulario Expresivo}

El análisis factorial para esta escala arroja un valor de $\mathrm{KMO}$ de 0.731, y en la prueba de esfericidad de Bartlett se obtiene una correlación significativa entre las variables (chi cuadrado de 753.9; $p<0.0001)$. Originalmente el análisis arrojó 6 factores, que explicaban el $66.3 \%$ de la varianza. Con el objetivo de reducir la cantidad de factores y que a su vez éstos incluyeran todos los reactivos, se fueron probando distintas soluciones, hasta llegar a 2 factores (los que contenían los pesos factoriales más altos de los reactivos), los cuales explican un $37.4 \%$ de la varianza en las respuestas. La Tabla 5 muestra dichos factores con el nombre propuesto, el número de ítems que consideran y el porcentaje de varianza explicada.

El Factor 1 agrupa 10 reactivos y se refiere al uso de un vocabulario inicial. El Factor 2 considera 9 ítems y hace alusión al uso de un vocabulario relacionado con rutinas diarias, reglas sociales y juegos. En síntesis, puede apreciarse que los factores que agrupan a los ítems se basan en el grado de dificultad de las palabras.

\section{Análisis factorial para Escala Gestos Totales}

El análisis factorial para esta escala arroja un valor de $\mathrm{KMO}$ de 0.806 , y en la prueba de esfericidad de Bartlett se obtiene una correlación significativa entre las variables (chi cuadrado de 1734.8; $p$ < 0.0001 ). Originalmente el análisis arrojó 12 factores, que explicaban el $66 \%$ de la varianza. Con el objetivo de reducir la cantidad de factores y que a su vez éstos incluyeran todos los reactivos, se fueron probando distintas soluciones, hasta llegar a 3 factores (que contenían los pesos factoriales más altos de los reactivos), los cuales explican un $35.2 \%$ de la varianza en las respuestas. La Tabla 5 muestra dichos factores con el nombre propuesto, el número de ítems que consideran y el porcentaje de varianza explicada.

El Factor 1 agrupa 10 reactivos y se refiere a la realización de gestos tempranos o iniciales. El Factor 2 considera 25 ítems y hace alusión al uso de gestos más elaborados y acciones de juego con un otro. Por último, el Factor 3 incluye 2 reactivos y alude a gestos faciales interactivos con otro. En síntesis, los factores que agrupan a los ítems se basan en el grado de dificultad o elaboración de los gestos y acciones.

\section{Análisis de validez de constructo de la versión abreviada del Inventario CDI}

\section{Correlaciones entre las subescalas y escalas}

En las Tablas 6 a 8 se aprecian las correlaciones entre las subescalas, al interior de cada una de ellas (sin considerar la primera escala, la cual no se divide en subescalas), de los CDI respondidos por los padres. Se puede observar que las distintas subescalas de las 3 escalas analizadas, presentan correlaciones significativas entre ellas (con un $p$ $<0.001$ ). Las cuatro subescalas de la Escala de Vocabulario Comprensivo presentan correlaciones altas entre sí, revelando que efectivamente se vinculan altamente a aspectos comprensivos del lenguaje. Las dos subescalas de la Escala de Vocabulario Expresivo presentan una correlación moderada entre ellas, indicando su vinculación a aspectos expresivos del lenguaje.

De la Escala de Gestos Totales, se da una correlación medio alta entre las subescalas 1 y 2 , lo cual muestra que ambas subescalas se vinculan con 


\section{TABLA 5}

Análisis Factorial reactivos Escalas Vocabulario Comprensivo, Vocabulario Expresivo y Gestos Totales, versión abreviada

\begin{tabular}{llcc}
\hline \multicolumn{1}{c}{ Escala } & \multicolumn{1}{c}{ Factores } & o de reactivos & \% varianza explicada \\
\hline \multirow{2}{*}{$\begin{array}{ll}\text { Vocabulario } \\
\text { Comprensivo }\end{array}$} & 2. Vocabulario inicial cotidiano & 32 & $7.068 \%$ \\
$\left(^{*}\right)$ & 2. Vocabulabulario inicial, menos cotidiano & 41 & $9.119 \%$ \\
& 4. Vocabulario avanzado, cotidiano & 36 & $9.852 \%$ \\
& TOTAL & 49 & $10.513 \%$ \\
& 1. Vocabulario inicial & 158 & $36.6 \%$ \\
Vocabulario & 2. Vocabulario relacionado con rutinas diarias, reglas socia- & 10 & $16.518 \%$ \\
Expresivo & les y juegos & 9 & $20.858 \%$ \\
& TOTAL & 19 & $37.4 \%$ \\
& 1. Primeros gestos & 10 & $10.416 \%$ \\
Gestos & 2. Gestos y acciones & 25 & $18.562 \%$ \\
Totales $(* *)$ & 3. Gestos faciales interactivos & 2 & $6.204 \%$ \\
& TOTAL & 37 & $35.2 \%$ \\
\hline
\end{tabular}

(*) Algunos reactivos no pudieron agruparse en ninguno de estos factores. Por sus características, en el instrumento final se incluyeron dentro de la subescala correspondiente al Factor 4.

(**) Hay un reactivo que no es agrupado en ninguno de estos factores. Por sus características, en el instrumento final se incluyó dentro de la subescala correspondiente al Factor 2.

Fuente: elaboración propia con base en Foster et al. (1994).

aspectos relacionados con los gestos. La subescala 3 en cambio presenta una baja correlación con la subescala 2, y no hay una correlación significativa con la subescala 1 , lo cual indica que esta subescala evalúa aspectos diferentes.

$\mathrm{Al}$ analizar las 4 escalas que conforman el CDI (véase Tabla 9), se puede apreciar que éstas presentan correlaciones significativas entre sí. Se observa una alta correlación entre Comprensión de Primeras Frases y Vocabulario Comprensivo, lo cual indica que ambas escalas evalúan aspectos relacionados con la comprensión del lenguaje. Se obtienen en cambio correlaciones moderadas entre Vocabulario Expresivo, y las dos escalas que refieren a aspectos comprensivos, lo cual indica que aunque todas refieren a aspectos del lenguaje, también se considera la diferencia entre lo comprensivo y lo expresivo. Por último, la escala de Gestos Totales presenta correlaciones medias altas con las demás escalas. Todo lo anterior indica que dichas escalas se vinculan a un mismo constructo (lenguaje) que es el que instrumento pretende evaluar.

\section{Correlaciones entre padres y educadoras}

Posteriormente, se llevó a cabo un análisis de correlaciones padres-educadoras entre las 4 escalas de la versión abreviada del instrumento, como otra evidencia de la validez de constructo del mismo. Contrariamente a lo esperado, no hay correlaciones estadísticamente significativas entre lo evaluado por los padres y lo evaluado por las educadoras, en las escalas de Comprensión de Primeras Frases, Vocabulario Comprensivo y Vocabulario Expresivo. Solo se observa una correlación moderada en la escala de Gestos Totales $(r=0.279 ; p=0.001)$. 
TABLA 6

Intercorrelaciones entre las 4 subescalas de la Escala de Vocabulario Comprensivo

\begin{tabular}{|c|c|c|c|c|c|}
\hline & & Subesc_2.1_corr & Subesc_2.2_corr & Subesc_2.3_corr & Subesc_2.4_corr \\
\hline \multirow[t]{3}{*}{ Subesc_2.1_corr } & Correlación de Pearson & 1 & $0.669(* *)$ & $0.697(* *)$ & $0.707(* *)$ \\
\hline & Sig. (bilateral) & & 0.000 & 0.000 & 0.000 \\
\hline & $N$ & & 130 & 130 & 130 \\
\hline \multirow[t]{3}{*}{ Subesc_2.2_corr } & Correlación de Pearson & & 1 & $0.804(* *)$ & $0.807(* *)$ \\
\hline & Sig. (bilateral) & & & 0.000 & 0.000 \\
\hline & $\mathrm{N}$ & & & 130 & 130 \\
\hline \multirow[t]{3}{*}{ Subesc_2.3_corr } & Correlación de Pearson & & & 1 & $0.789(* *)$ \\
\hline & Sig. (bilateral) & & & & 0.000 \\
\hline & $N$ & & & & 130 \\
\hline \multirow[t]{3}{*}{ Subesc_2.4_corr } & Correlación de Pearson & & & & 1 \\
\hline & Sig. (bilateral) & & & & \\
\hline & $\mathrm{N}$ & & & & \\
\hline
\end{tabular}

** La correlación es significativa al nivel .01 (bilateral).

Fuente: elaboración propia.

TABLA 7

Intercorrelaciones entre las 2 subescalas de la Escala de Vocabulario Expresivo

\begin{tabular}{|c|c|c|c|}
\hline & & Subesc_3.1_corr & Subesc_3.2_corr \\
\hline \multirow{3}{*}{ Subesc_3.1_corr } & Correlación de Pearson & 1 & $0.464(* *)$ \\
\hline & Sig. (bilateral) & & 0.000 \\
\hline & $\mathrm{N}$ & & 129 \\
\hline \multirow{3}{*}{ Subesc_3.2_corr } & Correlación de Pearson & & 1 \\
\hline & Sig. (bilateral) & & \\
\hline & $\mathrm{N}$ & & \\
\hline
\end{tabular}

** La correlación es significativa al nivel .01 (bilateral).

Fuente: elaboración propia. 
TABLA 8

Intercorrelaciones entre las 3 subescalas de la Escala Gestos Totales

\begin{tabular}{|c|c|c|c|c|}
\hline & & Subesc_4.1_corr & Subesc_4.2_corr & Subesc_4.3_corr \\
\hline \multirow{3}{*}{ Subesc_4.1_corr } & Correlación de Pearson & 1 & $0.508(* *)$ & 0.145 \\
\hline & Sig. (bilateral) & & 0.000 & 0.101 \\
\hline & $\mathrm{N}$ & & 130 & 130 \\
\hline \multirow{3}{*}{ Subesc_4.2_corr } & Correlación de Pearson & & 1 & $0.193(*)$ \\
\hline & Sig. (bilateral) & & & 0.028 \\
\hline & $\mathrm{N}$ & & & 130 \\
\hline \multirow{3}{*}{ Subesc_4.3_corr } & Correlación de Pearson & & & 1 \\
\hline & Sig. (bilateral) & & & \\
\hline & $\mathrm{N}$ & & & \\
\hline
\end{tabular}

* La correlación es significante al nivel .05 (bilateral).

** La correlación es significativa al nivel .01 (bilateral).

Fuente: elaboración propia.

TABLA 9

Intercorrelaciones entre las 4 escalas del CDI, versión abreviada

\begin{tabular}{|c|c|c|c|c|c|}
\hline & & $\begin{array}{l}\text { Comprensión } \\
\text { primeras frases }\end{array}$ & $\begin{array}{l}\text { Vocabulario } \\
\text { comprensivo }\end{array}$ & $\begin{array}{c}\text { Vocabulario } \\
\text { expresivo }\end{array}$ & $\begin{array}{l}\text { Gestos } \\
\text { totales }\end{array}$ \\
\hline \multirow[t]{3}{*}{ Comprensión primeras frases } & Correlación de Pearson & 1 & $0.624(* *)$ & $0.236(* *)$ & $0.498(* *)$ \\
\hline & Sig. (bilateral) & & 0.000 & 0.007 & 0.000 \\
\hline & $\mathrm{N}$ & & 130 & 129 & 130 \\
\hline \multirow[t]{3}{*}{ Vocabulario comprensivo } & Correlación de Pearson & & 1 & $0.394(* *)$ & $0.564(* *)$ \\
\hline & Sig. (bilateral) & & & 0.000 & 0.000 \\
\hline & $N$ & & & 129 & 130 \\
\hline \multirow[t]{3}{*}{ Vocabulario expresivo } & Correlación de Pearson & & & 1 & $0.468(* *)$ \\
\hline & Sig. (bilateral) & & & & 0.000 \\
\hline & $N$ & & & & 129 \\
\hline \multirow[t]{3}{*}{ Gestos totales } & Correlación de Pearson & & & & 1 \\
\hline & Sig. (bilateral) & & & & \\
\hline & $N$ & & & & 130 \\
\hline
\end{tabular}

** La correlación es significativa al nivel .01 (bilateral).

Fuente: elaboración propia. 
Correlaciones entre CDI padres y educadoras, con Bayley III

Finalmente se correlacionaron las evaluaciones realizadas por padres y educadoras respecto del lenguaje del niño (considerando las escalas Comprensión de Primeras Frases, Vocabulario Comprensivo y Vocabulario Expresivo), con las subescalas de lenguaje comprensivo y expresivo así como la escala total de lenguaje del Bayley III. En los análisis realizados se aprecia la ausencia de correlaciones significativas, en todos los análisis.

Correlaciones entre padres y educadoras a los 18 meses de edad de los niños

$\mathrm{Al}$ repetir este análisis con los datos de una segunda aplicación a los 18 meses de los niños ( $N=$ 122), se aprecia una correlación significativa entre padres y educadoras en la escala de Vocabulario Expresivo $(r=0.266 ; p=0.003)$, no así en las restantes escalas.

Correlaciones entre CDI padres y educadoras, con Bayley III a los 18 meses de edad de los niños

$\mathrm{Al}$ analizar las relaciones entre las percepciones de padres y educadoras con el Bayley III $(\mathrm{N}=55)$, se observan correlaciones significativas entre la escala de Vocabulario Expresivo del CDI y la subescala de lenguaje expresivo del Bayley III, tanto en los padres $(r=0.526$; $p=0.000)$ como en las educadoras $(r=0.373 ; p=0.005)$. Igualmente correlacionan significativamente esta escala del CDI con la escala de Lenguaje del Bayley III, en padres $(r=0.566 ; p=0.000)$ y educadoras $(r=$ $0.285 ; p=0.035)$.

En cuanto al lenguaje comprensivo, sólo se da una correlación inversamente significativa entre al escala de Vocabulario Comprensivo del CDI en los padres y la escala de Lenguaje del Bayley III $(r$ $=-0.296 ; p=0.028)$. Es decir, mientras menor desarrollo del lenguaje reporta el Bayley III, mayor percepción de presencia de comprensión de vocabulario reportan tener los padres respecto de sus hijos.

\section{Discusión}

El inventario del Desarrollo de Habilidades Comunicativas de MacArthur-Bates (CDI), versión W \& $\mathrm{G}$ ("Primeras palabras y gestos"), es un instrumento cuyo objetivo es evaluar el desarrollo de habilidades comunicativas en niños y niñas, a través del reporte de un cuidador significativo. Consta de 941 reactivos, distribuidos en 6 escalas, las cuales a su vez se subdividen en subescalas relacionadas con categorías semánticas.

En este estudio, el CDI fue aplicado a una muestra de 130 niños chilenos entre 11 y 15 meses de edad, pertenecientes a un NSE bajo y de familias en riesgo psicosocial. El inventario fue respondido por un familiar significativo del niño (principalmente la madre) y por el personal de la sala cuna (educadora de párvulos o técnica auxiliar de párvulos).

En los análisis realizados, primero se procedió a evaluar la confiabilidad del instrumento, a través del coeficiente alfa de Cronbach. Tanto el instrumento respondido por los padres así como por las educadoras, obtuvieron buenos índices de consistencia interna, oscilando entre 0.885 y 0.990 , aunque cabe considerar que no todas las escalas arrojaron información por el bajo índice de respuestas (versión educadoras).

Luego, se realizó el análisis de los reactivos a través del software ITEMAN y con las respuestas entregadas por los padres, tras lo cual se seleccionaron solo aquellos reactivos que presentaban buenas características psicométricas (con excepción de la escala de vocabulario expresivo, donde además se incluyeron reactivos regulares de modo que la escala no quedara demasiado reducida). La versión abreviada del instrumento quedó compuesta por 241 reactivos. También se modificó el número de escalas, dejando 4 escalas finales de las 6 originales que componían el CDI: Comprensión de Primeras Frases, Vocabulario Comprensivo, Vocabulario Expresivo y Gestos Totales. A su vez, con excepción de la primera escala que no consideraba subescalas, se modificaron las subescalas originales que componían las escalas, a través de la información entregada por el análisis factorial. En la versión 
abreviada, las escalas quedaron compuestas por 4,2 y 3 subescalas, respectivamente. A diferencia de la versión original donde la distribución en subescalas de los reactivos obedece a criterios de categorías semánticas, en la versión abreviada la distribución obedece principalmente a características relacionadas con el grado de dificultad de las palabras o gestos.

Posteriormente, se analizó la confiabilidad de la versión abreviada del CDI, nuevamente con el coeficiente alfa de Cronbach, observándose muy poca variación respecto a los primeros resultados, y manteniéndose una buena consistencia interna, dentro de un rango de 0.841 a 0.982 para los padres, y 0.837 a 0.981 para las educadoras. Cabe destacar que, con la versión abreviada del CDI, sí se pudo obtener información de confiabilidad para todas las escalas.

Estudios previos de confiabilidad del CDI en una muestra de 659 niños americanos, arrojaron un coeficiente alfa de Cronbach de 0.95 para Vocabulario Comprensivo y 0.96 para Vocabulario Expresivo. En una muestra de 778 niños mexicanos, se obtuvo un coeficiente alfa de Cronbach de 0.94 para Vocabulario Comprensivo, 0.94 para Vocabulario Expresivo, y 0.89 para Gestos Totales. En comparación con estos datos, se puede observar que los índices de confiabilidad obtenidos para la versión abreviada del CDI en la muestra de niños chilenos, pese a ser mucho más reducida en número a las muestras americanas y mexicanas, arroja mejores índices de confiabilidad en las escalas de Vocabulario Comprensivo y Gestos Totales. No es el caso del Vocabulario Expresivo, lo cual se debe probablemente al número reducido de reactivos con los cuales quedó conformada la escala (19 vs. 428$)$, pero se optó por una escala compuesta por menos reactivos pero con buenas propiedades psicométricas, lo cual además irá en beneficio de la prueba ya que requiere un tiempo de aplicación mucho menor, lo cual tiene una serie de beneficios respecto de la motivación y actitud de los adultos que deban contestarla, especialmente en aquellos casos de las educadoras, quienes deben contestar evaluaciones de varios niños, aumentando por tanto la credibilidad de sus respuestas.
En seguida, se procedió a obtener evidencias de la validez de constructo de la versión abreviada del CDI. En los análisis realizados se aprecian, en general, correlaciones entre moderadas a altas, entre las subescalas que componen las escalas, así como entre las escalas entre sí, lo cual es similar a lo reportado por los análisis con muestra americana y mexicana, y arroja evidencias de la validez correlacional del CDI.

En cambio, al correlacionar la percepción del lenguaje del niño entre padres y educadoras, con el fin de tener información sobre la validez convergente, no se obtuvieron correlaciones significativas, excepto algunas correlaciones moderadas en la escala de Gestos Totales y luego en la escala de Vocabulario Expresivo, a los 18 meses de edad de los niños.

Tampoco se obtuvieron correlaciones significativas al analizar las escalas del CDI versión abreviada de padres y de educadoras, con las subescalas de lenguaje del Bayley III. Al repetir los análisis con datos obtenidos a los 18 meses de edad de los niños, se observan correlaciones moderadas en las educadoras y altas en los padres entre la escala de Vocabulario Expresivo del CDI con la subescala de lenguaje expresivo y con la escala de Lenguaje del Bayley III, y correlaciones moderadas inversamente significativas entre Vocabulario Comprensivo del CDI padres con la escala de Lenguaje del Bayley III.

Ello coincide relativamente con otros estudios realizados tanto en muestra americana como mexicana, que aportan evidencias de validez convergente entre moderada y alta, para la parte de vocabulario expresivo del CDI.

Los resultados obtenidos en este estudio hacen pensar que la versión abreviada propuesta del CDI presenta evidencias de validez correlacional, tanto a nivel de subescalas como de las escalas entre sí, por tanto el inventario es consistente en cuanto al constructo que está evaluando. Pero los datos obtenidos para la validez convergente muestran que lo evaluado por los padres difiere de lo evaluado por las educadoras (a excepción de la escala de Gestos Totales), y ambos a su vez difieren de la evaluación del Bayley III. Ello puede deberse a que padres y educadoras tenían diferencias significati- 
vas en cuanto a su comprensión y/o compromiso con la tarea, o a que su conocimiento del niño(a) es diferente debido a que lo observan en situaciones cotidianas distintas. A su vez, el que ellos difieran de los resultados arrojados por el Bayley III, puede hacer pensar que se podrían estar evaluando constructos distintos (por ejemplo, la percepción que los adultos tienen del nivel de lenguaje logrado por el niño, que puede verse influida por una serie de sesgos tanto cognitivos como afectivos). También debe considerarse la dificultad de evaluar el rendimiento del niño a través de la observación de personas cercanas, a edades tan tempranas cuando recién la habilidad está comenzando a desarrollarse y manifestarse. Esos resultados son interesantes y plantean preguntas para futuros investigadores.

Si consideramos que el Bayley III es un instrumento aplicado al niño por un evaluador experto - entrenado- podríamos suponer entonces que es el instrumento el que nos está entregando una versión más "objetiva" o menos sesgada del lenguaje del niño, en sus aspectos de vocabulario comprensivo y expresivo. El CDI, en cambio, no evalúa de manera directa el lenguaje propiamente tal, sino la percepción (subjetiva) que tienen padres y educadoras, respecto del lenguaje del niño.

La percepción de las capacidades de otro por un evaluador no entrenado, siempre estará sujeta al efecto de otras variables, y en esta muestra específica, que se caracteriza por un bajo NSE, factores de riesgo psicosocial, estrés laboral y malas condiciones de trabajo en las educadoras, etc., influye en que la percepción sea más sesgada. Estudios previos han demostrado que en poblaciones chilenas de estas características psicosociales, los padres tienden a sobredimensionar los puntajes de sus hijos (sesgo positivo), mientras que en las educadoras tienden a subvalorar los rendimientos y capacidades de los niños (sesgo negativo). La especial atención que se ha prestado al lenguaje y desarrollo de los niños por parte de los planes de gobierno, hace que el sesgo aumente (lo cual no es el caso del aspecto gestual, donde sí se dan correlaciones positivas entre padres y educadoras; lamentablemente no hay un escala del Bayley que evalúe esto, para poder compararlo).
Cabe mencionar que en este estudio se tomaron las medidas habituales para que los evaluados (padres y educadoras) entendieran bien las instrucciones y pudieran contestar de buena forma el instrumento, cuidando las instrucciones y supervisión por parte de los evaluadores que aplicaron el CDI (a través de un entrenamiento previo y homogeneización de la aplicación, con el propósito de controlar potenciales variables del evaluador). Pero no se tomaron medidas adicionales previniendo un posible sesgo, ya que no se esperaba encontrar diferencias. Se recomienda, por tanto, considerar esta variable en futuros estudios, lo cual podría hacerse por ejemplo acompañando la aplicación del instrumento de una observación en terreno previa del niño para luego apoyar al evaluado en sus respuestas al CDI. Otra alternativa es dar por descontado que el sesgo estará presente, y comparar a los niños por su delta de crecimiento entre una medición y otra, más que en términos de los puntajes alcanzados.

Al repetir la evaluación a los 18 meses de edad de los niños, se aprecia que la escala de Vocabulario Expresivo presenta evidencias de validez convergente, tanto en la percepción de padres y educadoras entre sí, así como en relación al Bayley III, coincidiendo con otros estudios. Ello puede explicarse en el hecho de que al comenzar a aumentar el vocabulario expresivo del niño, aumenta la evidencia directa sobre este aspecto y sería más fácil llegar a una percepción compartida entre evaluadores expertos y no expertos. No sería, en cambio, el caso del lenguaje comprensivo, donde las percepciones o atribuciones que realiza en adulto sobre éste siguen posiblemente viéndose influidas por diversos sesgos, al no contar con evidencia directa.

Otro factor que influye es el motivacional. La versión original del CDI es bastante larga y extensa, afectando especialmente las respuestas de las educadoras, las cuales deben responder sus evaluaciones sobre varios niños, en cambio los padres contestan una sola evaluación relativa a su hijo. También se debe considerar como factor adicional, el nivel de lenguaje de padres y educadoras el cual influye en su comprensión y respuestas al CDI. Ya 
el hecho de poder contar con una versión abreviada del instrumento podrá aportar a subsanar el tema motivacional. También es recomendable distribuir en el tiempo las evaluaciones realizadas por las educadoras, de modo que no se acumulen varias evaluaciones de distintos niños en un tiempo muy corto. Otras alternativas son también recomendables, como por ejemplo incorporar a padres y educadoras respecto a la relevancia del estudio y la importancia de contestar de manera lo más fidedigna posible los instrumentos, retribuirles su cooperación con material relevante para su quehacer o poderles informar luego los resultados, acompañados de sugerencias. Respecto a las dificultades de lenguaje, aunque este instrumento está descrito como autoaplicable, un acompañamiento directo y continuo por parte del evaluador(a) se hace indispensable.

En síntesis, este análisis ha permitido proponer una versión abreviada del CDI (forma W \& G) bastante más reducida que la versión original, lo cual irá en beneficio de la motivación y actitud de los adultos que deban contestarla. Su confiabilidad es buena, presenta evidencias de validez de constructo (correlacional y factorial), sus reactivos presentan buenas propiedades psicométricas y por tanto permitirán discriminar adecuadamente el nivel de desarrollo de una muestra (por ejemplo el efecto de distintas intervenciones). Cabe considerarse sí que la muestra empleada en este estudio fue bastante homogénea, por tanto su uso de manera más generalizada debe realizarse con cautela y nuevos estudios se hacen recomendables. Si, en cambio, se desea conocer el tamaño del vocabulario comprensivo o expresivo de los niños, se recomienda usar la versión original, acompañada de otro tipo de evaluaciones u observaciones.

Al considerar que lo que el CDI evalúa es la percepción del adulto sobre las capacidades lingüísticas del niño, se recomienda no tomarlo por sí sólo como una medida del lenguaje real de éste. Podrían, por ejemplo, promediarse las percepciones de distintos adultos, o complementar con otro tipo de evaluación. Se podrían, además, hacer estudios de validez convergente con otros instrumentos que evalúen percepción del adulto sobre las capa- cidades del niño, y quizá usarse exitosamente para evaluar los efectos de intervenciones orientadas a disminuir los sesgos hacia las capacidades de los niños.

\section{Conclusiones}

La versión abreviada del CDI (forma W \& G) evalúa la percepción del adulto respecto al desarrollo de habilidades comunicativas en niños y niñas entre 8 y 18 meses de edad, a través del reporte de un cuidador significativo. Después de los 18 meses, y considerando el gran desarrollo que tiene el lenguaje en esta edad, se recomienda el uso de la segunda forma del CDI (W \& S). La versión abreviada se compone de 241 reactivos, y entrega puntajes divididos en 4 escalas, que varían entre 0 y 19 puntos a 0 y 165 puntos, según la escala. La consistencia interna, usando el coeficiente alfa, es adecuada; presenta evidencias de validez de constructo (correlacional) y sus reactivos presentan buenas propiedades psicométricas. Se recomienda su uso principalmente en estudios que busquen discriminar adecuadamente el nivel de desarrollo de una muestra o estudiar el efecto de distintas intervenciones. Aunque son necesarios estudios posteriores de esta versión abreviada en muestras de mayor tamaño y heterogeneidad, así como en relación con otros instrumentos que evalúan lenguaje en esta edad con el fin de investigar más a fondo su validez, presenta como ventaja el ser una versión más acotada del instrumento original, lo cual reduce su tiempo de respuesta, afecta menos la motivación de la persona que contesta, genera menos cansancio y constituye, por tanto, una herramienta valiosa para futuros estudios.

\section{Referencias}

Acredolo, L. \& Goodwyn, S. (1985). Symbolic gesturing in language development: A case study. Human Development, 28(1), 40-49.

Acredolo, L. \& Goodwyn, S. (1988). Symbolic gesturing in normal infants. Child Development, 59(2), $450-456$. 
Bates, E., Benigni, L., Bretherton, I., Camaioni, L. \& Volterra, E. (1979). The emergence of symbols: Cognition and communication in infancy. San Diego: Academic Press.

Bates, E., Bretherton, I. \& Snyder, L. (1988). From first words to grammar: Individual differences and dissociable mechanisms. New York: Cambridge University Press.

Bates, E., Camaioni, L. \& Volterra, V. (1975). The acquisition of performatives prior to speech. Merril-Palmer Quarterly, 21 (3), 205-226.

Bayley, N. (2006). Bayley Scales of Infant and Toddler Development (3rd ed.). San Antonio, TX: The Psychological Corporation.

Fenson, L., Dale, P. S., Reznick, J. S., Bates, E., Thal, D. J., Pethick, S. J. et al. (1994). Variability in early communicative development. Monographs of the Society for Research in Child Development, 59(5), 1-190.

Fenson, L., Thal, D. J. \& Bates, E. (1990). Normed values for the "Early Language Inventory" and three associated parent report forms for language assessment (Technical Report). San Diego: San Diego State University.

Jackson-Maldonado, D., Thal, D., Fenson, L., Marchman, V., Newton, T. \& Conboy, B. (2003). Mac-
Arthur Inventarios del desarrollo de habilidades comunicativas. Baltimore: Brookes Publishing.

Nunnally J. C. (1978). Psychometric Theory. New York: McGraw-Hill.

Piaget, J. (1962). Play, dreams and imitation in childhood. New York: W.W. Norton.

Rescorla, L. \& Goossens, M. (1992). Symbolic play development in toddlers with expressive specific language impairment. Journal of Speech and Hearing Research, 6, 1290-1302.

Terrell, B. \& Schwartz, R. (1988). Objects transformations in the play of language-impaired children. Journal of Speech and Hearing Research, 53, 459-466.

Terrell, B., Schwartz, R., Prelock, P. \& Messick, C. (1984). Symbolic play in normal and languageimpaired children. Journal of Speech and Hearing Research, 27, 424-429.

Thal, D. \& Tobias, S. (1993). Communicative gestures in children with delayed onset of oral expressive vocabulary. Journal of Speech and Hearing Research, 35, 1281-1289.

Uzgiris, I. \& Hunt, J. (1975). Assessment in infancy: Ordinal scales of psychological development. Champaign: University of Illinois Press. 